\title{
Predicting the binding type of compounds on the 4 adenosine receptors using proteochemometric models
}

\author{
Olaf van den Hoven ${ }^{*}$, Gerard van Westen, Andreas Bender \\ From 5th German Conference on Cheminformatics: 23. ClC-Workshop \\ Goslar, Germany. 8-10 November 2009
}

\begin{abstract}
Aim
The use of in silico models could save a lot of money, time and resources in the laboratories when in search for potent binding candidates of designated receptors. In this paper a proteochemometric model was developed that is able to distinguish between full agonist, agonist and antagonist across all 4 Adenosine receptors (A1, $\mathrm{A} 2 \mathrm{~A}, \mathrm{~A} 2 \mathrm{~B}$ and $\mathrm{A} 3$ ).
\end{abstract}

\section{Experimental}

The software program Pipeline Pilot was used to create a proteochemometric model containing 2 SVMs [1]. The first SVM model was built to predict binding activity, the second SVM model to predict binding type activity. For the first model an in house database, Glida and part of the Starlite database were used. The pKi values in these databases were then capped to create an active and inactive class. For the second model a classification in full agonist, agonist and antagonist derived from the Glida database was used. Different Proteinfingerprints (ProtFPs) were tested. These FPs consisted of amino acid residues at important binding positions according to two entropy analysis (TEA) and crystal structure analysis [2]. The quality of the model was determined by MCC, specificity, sensitivity, predictive ability (Q2) and the correlation coefficient (R2). The Tanimoto similarity coefficient was used to visualize the degree of average and minimal similarity of the three binding type groups.

\section{Results}

In continuous models FPs having a combination of TEA and crystal structure information scored the best $(\mathrm{Q} 2=$ 0.6683 ), but overall scores were not high enough to benefit from a continuous model. A categorical model was thus build and it was able to distinguish between agonist (99\% correctly predicted) and antagonist (98\% correct) and partially full agonists (63\% correct) with a specificity of 0.85 , a sensitivity of 0.83 and a MCC of 0.69 . The Tanimoto coefficient showed that indeed antagonist and full agonist were the least similar, where agonist show more resemblance to the full agonist. The model finally predicted 2 novel full agonistic compounds in our in house database, being GIFT136 and GIFT589.

\section{Conclusion}

A model that is able to distinguish between binding types was successfully created.

Published: 4 May 2010

\section{References}

1. Kontijevskis A: J Chem Inf Model 2008, 48(9):1840.

2. Ye K: Proteins 2006, 63(4):1018.

\section{doi:10.1186/1758-2946-2-S1-P16}

Cite this article as: van den Hoven et al:: Predicting the binding type of compounds on the 4 adenosine receptors using proteochemometric models. Journal of Cheminformatics 2010 2(Suppl 1):P16. 